Correction: Proteomic screening identifies the zonula occludens protein ZO-1 as a new partner for ADAM12 in invadopodia-like structures

\author{
Bassil Dekky ${ }^{1}$, Michael Ruff ${ }^{1,2, *}$, Dominique Bonnier ${ }^{1, *}$, Vincent Legagneux ${ }^{1}$ and \\ Nathalie Théret ${ }^{1}$ \\ ${ }^{1}$ Univ Rennes, Inserm, EHESP, Irset (Institut de recherche en santé, environnement et travail)-UMR_S1085, Rennes, France \\ ${ }^{2}$ Present address: Institute of Biology Valrose (iBV) CNRS UMR7277 - INSERM U1091 - UNS University Nice Sophia Antipolis, \\ Nice, France \\ * These authors contributed equally to this work \\ Published: November 06, 2018
}

Copyright: Dekky et al. This is an open-access article distributed under the terms of the Creative Commons Attribution License 3.0 (CC BY 3.0), which permits unrestricted use, distribution, and reproduction in any medium, provided the original author and source are credited.

This article has been corrected: The following sentence has been added to the first paragraph in the Material and Methods section: 'Protein digestion and mass spectrometry analyses were performed by the Proteomics Platform of the CHU de Québec Research Center (Quebec, Qc,Canada).'

\title{
Proteomics analyses
}

Immunoprecipitation of proteins associated with ADAM12L were performed using extracts from ADAM12Loverexpressing MCF10A cells and immunoprecipitation using Rabbit IgG was included as control. Proteins from immunoprecipitates were denaturated and size-separated by sodium dodecyl sulfate-polyacrylamide gel electrophoresis (SDSPAGE). After cutting gel tracks in 10 bands, in-gel digestion of proteins was performed with trypsin and resulting peptides were injected into a capillary HPLC system coupled to a mass spectrometer via a nanospray ionization source (ES MS/MS). All MS/MS samples were analyzed using Mascot (Matrix Science, London, UK; version 2.4.1) and X! Tandem softwares. Mascot was set up to search the TAX_HomoSapiens_9606_20141128 database. X! Tandem was set up to search a subset of the TAX_HomoSapiens_9606_20141128 database. For protein identification, Scaffold software (version 4.4.1.1, Proteome Software Inc., Portland, OR) was used to validate MS/MS based peptide and protein identifications. Peptide identifications were validated when established at a probability greater than $86,0 \%$ to achieve an FDR less than $1,0 \%$ using the Scaffold Local FalseDiscoveryRate (FDR) algorithm. Protein identifications were validated when established at a probability greater than 99,0 \% probability to achieve an FDR less than 1,0\% and contained at least 2 identified peptides. Protein probabilities were assigned by the Protein Prophet algorithm [69]. Protein digestion and mass spectrometry analyses were performed by the Proteomics Platform of the CHU de Québec Research Center (Quebec, Qc,Canada).

Original article: Oncotarget. 2018; 9:21366-21382. https://doi.org/10.18632/oncotarget.25106 